\title{
Adolescent-onset anorexia nervosa: 18-year outcome
}

\author{
Elisabet Wentz, I. Carina Gillberg, Henrik Anckarsäter, Christopher Gillberg \\ and Maria Råstam
}

\section{Background}

The long-term outcome of anorexia nervosa is insufficiently researched.

\section{Aims}

To study prospectively the long-term outcome and prognostic factors in a representative sample of people with teenage-onset anorexia nervosa.

\section{Method}

Fifty-one people with anorexia nervosa, recruited by community screening and with a mean age at onset of 14 years were compared with 51 matched comparison individuals at a mean age of 32 years (18 years after disorder onset). All participants had been examined at ages 16 years, 21 years and 24 years. They were interviewed for Axis I psychiatric disorders and overall outcome (Morgan-Russell assessment schedule and the Global Assessment of Functioning)

\section{Results}

There were no deaths. Twelve per cent $(n=6)$ had a persisting eating disorder, including three with anorexia nervosa. Thirty-nine per cent of the anorexia nervosa group met the criteria for at least one psychiatric disorder. The general outcome was poor in $12 \%$. One in four did not have paid employment owing to psychiatric problems. Poor outcome was predicted by premorbid obsessive-compulsive personality disorder, age at onset of anorexia nervosa and autistic traits.

\section{Conclusions}

The 18-year outcome of teenage-onset anorexia nervosa is favourable in respect of mortality and persisting eating disorder.

\section{Declaration of interest}

None. Funding detailed in Acknowledgements.
Anorexia nervosa is one of the most severe psychiatric disorders found in adolescence. In most cases the teenager will gradually recover from the illness, but anorexia nervosa is also known for its severe complications, with chronicity and death being the two most feared consequences. In Steinhausen's review of all follow-up studies during the second half of the 20th century, chronicity occurred in one in five of all anorexia nervosa cases. ${ }^{1}$ There are only three published studies on anorexia nervosa that report long-term outcome. ${ }^{2-4}$ All three are based on severe cases of anorexia nervosa, with a sample of only tertiary referrals and/ or in-patients. The high mortality rates in these studies $(17 \%$, $18 \%$ and $16 \%$ respectively) corresponded in the study by Zipfel et al to a standardised mortality ratio of $9.8 .^{4}$ The occurrence of chronic anorexia nervosa in the three studies ranged from 8 to $25 \%$. Researchers have indicated that recovery after $10-12$ years is uncommon. ${ }^{2,5}$ However, a growing number of follow-up studies have focused on more homogeneous anorexia nervosa samples, i.e. only individuals with teenage-onset anorexia nervosa who seem to have a brighter future. Long-term studies (10 years or more) of adolescent-onset anorexia nervosa tend to report more favourable outcomes compared with other follow-up studies. $^{5-8}$

Since the mid-1980s our group has conducted the only controlled, prospective community-based study of adolescentonset anorexia nervosa. ${ }^{9}$ So far we have reported on the anorexia nervosa group and their matched controls on three occasions, at mean ages 16 years, 21 years and 24 years. There has been no attrition. In this paper we report our fourth study, 18 years after anorexia nervosa onset. We predicted a better general outcome and less psychiatric comorbidity compared with other long-term outcome studies based on in-patient data, and also that a comparison of general outcome and psychiatric comorbidity with the control group would reveal continuing difficulties in our community-based anorexia nervosa group in spite of the high rate of recovery from the eating disorder.

\section{Method}

\section{Original study (Study 1)}

In 1985 all 4291 individuals born in 1970 in the Swedish city of Göteborg were screened for anorexia nervosa in the eighth grade of junior high school using individual physical examinations in all cases, analysis of growth charts and an eating disorders questionnaire. ${ }^{10}$ Twenty-three girls and two boys constituted the total population of individuals born in 1970 who developed anorexia nervosa before the age of 18 years. These 25 cases correspond to a population prevalence of anorexia nervosa in those aged 17 years and under of $1.08 \%$ for girls and $0.09 \%$ for boys, and a population-corrected female:male ratio of 11.6:1. The details of the epidemiological study have been published previously. ${ }^{10,11}$ One girl agreed only to examination by the school health services, leaving 24 individuals ( 22 girls, 2 boys) for in-depth study. Because all the teenagers in this birth cohort were examined without their clothes on, had their growth charts scrutinised in detail and were followed up for at least a year by the same school nurse, and because $99.7 \%$ of them completed an anorexia nervosa screening questionnaire, we believe that we did not miss a single case of anorexia nervosa in this cohort.

The population group was pooled with another population screening sample of anorexia nervosa cases (26 girls, 1 boy), who were reported to the research team mainly by school health nurses and doctors during follow-up of the original population group. Almost all of these individuals were born in 1971-4. This sample was less comprehensive than the original one. We estimated that the population screening sample comprised about 
$60 \%$ of all anorexia nervosa cases in their birth cohort. The total examined anorexia nervosa group thus consisted of 51 cases (48 girls, 3 boys) who all met or had met the DSM-III-R and DSM-IV criteria for anorexia nervosa. ${ }^{12,13}$

\section{Comparison group}

For each of the 51 cases of anorexia nervosa the school nurse selected the same-sex classmate closest in age to the index child. The only exclusion criteria for individuals in the comparison group were a history of eating disturbances according to school nurses' reports and growth chart deviances from age 7 years and onwards.

In the original study all 102 individuals (51 individuals in the anorexia nervosa group and 51 individuals in the comparison group) and their mothers were seen by a psychiatrist (M.R.). Within the year of assessment in the original study all 51 individuals in the anorexia nervosa group fulfilled DSM-III-R and DSMIV criteria for anorexia nervosa, although at the precise time of examination 6 individuals in the anorexia nervosa group had bulimia nervosa and 6 further individuals at that time only partially fulfilled anorexia nervosa criteria and were diagnosed with eating disorder not otherwise specified (EDNOS) (Fig. 1). The individuals who had crossed over from anorexia nervosa to other eating disorders were not excluded from the original study or the subsequent follow-up studies.

A detailed developmental history was obtained for each child by interviewing the mother. The mothers were asked about eating disturbances from childhood onwards as well as current eating disorders. An evaluation of pregnancy, obstetrical and other medical records was made. On the basis of anonymised case notes on premorbid history, the 102 participants were assigned diagnoses of DSM-III-R personality disorders and autism-spectrum conditions by a senior psychiatrist (C.G.) who was masked to group status.

\section{Follow-up studies (Studies 2 and 3)}

All 102 individuals were examined again in Study 2 at a mean age of 21 years (6 years after anorexia nervosa onset), ${ }^{14,15}$ and in Study 3 at a mean age of 24 years ( 10 years after onset). ${ }^{7}$

\section{Study 4}

All 51 cases of anorexia nervosa were followed up in Study 4. Forty-five individuals agreed to a personal interview and five were interviewed over the telephone. In one case the mother did not want us to see her daughter, who had persisting anorexia nervosa, but agreed to a collateral interview with the semi-structured instruments used with the other participants. The 51 individuals in the comparison group all participated in Study 4 . All but three were interviewed in person; these three were interviewed over the telephone. The study was approved by the human ethics committee at the medical faculty of Göteborg University. The individuals participated voluntarily after informed consent was obtained.

\section{Measures}

The Structured Clinical Interview for DSM-IV Axis I Disorders (SCID-I) and the Morgan-Russell scales were used, ${ }^{16,17}$ by either E.W. or M.R. Assessment of overall outcome was based on the three measures of the Morgan-Russell scales with the later modification by the Russell group. ${ }^{3}$

(a) General outcome: categorised as good, intermediate or poor. A good outcome is a normal body weight - a body mass index (BMI) of $18.5 \mathrm{~kg} / \mathrm{m}^{2}$ or over - and normal menstruation; an intermediate outcome is normal or near-normal body weight or normal menstruation; and poor outcome is underweight and absent or scanty menstruation.

(b) Persistence of disorder: individuals with overeating or vomiting (weekly or more) were assigned to the poor outcome group even if they had normal weight and menstrual cycles. $^{3}$

(c) Morgan-Russell averaged scale score: this composite score summarises outcome data on body weight, diet restriction, menstruation, attitude to sex and menstruation, social relationships, relationship with family and mental state.

The Morgan-Russell instrument is currently the best-validated instrument in anorexia nervosa outcome research. ${ }^{1}$

\section{Global Assessment of Functioning}

Each participant's current social and occupational functioning was rated on the Global Assessment of Functioning (GAF) scale by E.W. or M.R. according to the DSM-IV. ${ }^{13}$ A GAF score of 60 or below was considered to represent poor psychosocial functioning.

\section{Full symptomatic recovery}

As proposed by Strober et $a l^{5}$ full recovery refers to patients who have been free of all criterion symptoms of anorexia nervosa or bulimia nervosa for not less than 8 consecutive weeks. In this study the criterion was freedom from the symptoms for more than 6 months. The study by Strober et al had good interrater reliability. ${ }^{5}$

\section{Psychiatric treatment}

Psychotherapy was defined in our earlier studies as child or adult psychiatric treatment given on eight occasions or more on an out-patient basis or in-patient treatment. The treatment received was either family or individual psychotherapy based on psychodynamic interventions. The most common form of treatment used was conjoint family therapy (according to

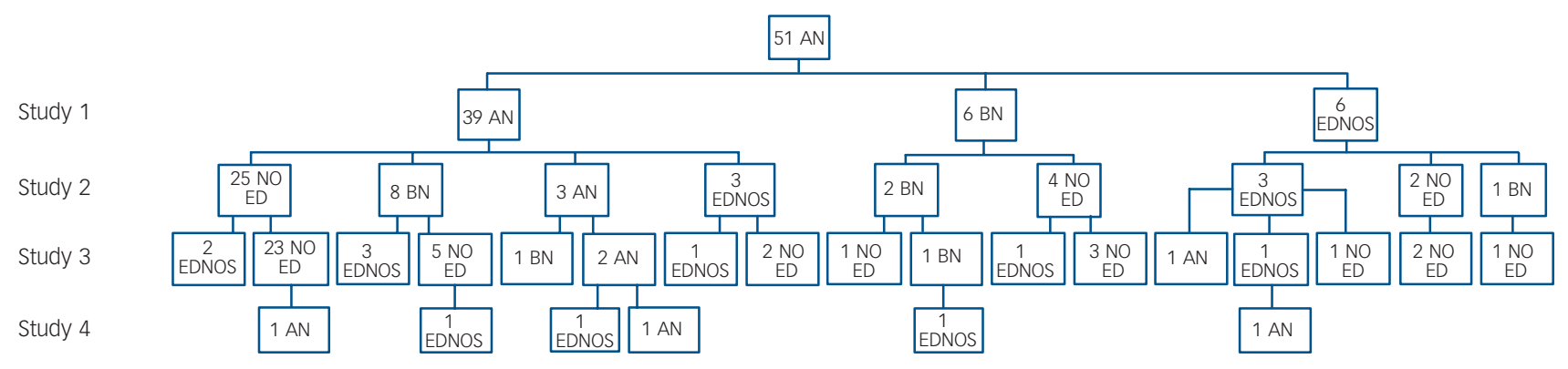

Fig. 1 Eating disorders in the four studies of this cohort. In Study 4, only individuals with a persisting eating disorder are presented. AN, anorexia nervosa; BN, bulimia nervosa; EDNOS, eating disorder not otherwise specified; NO ED, no eating disorder. 
descriptions by the individuals and their mothers and supported by medical and psychiatric records). Most of those who had received family therapy had also been given the opportunity of individual psychotherapy. In 1985, at the time of the original study, there was no specialised unit for children and adolescents with eating disorders in the city of Göteborg (a specialised unit opened in 1994). Families with a child with anorexia nervosa primarily sought treatment at child psychiatric and paediatric clinics, and in some cases only gynaecologists or general practitioners were consulted.

\section{Statistical analysis}

Scale scores were not normally distributed and therefore mainly non-parametric tests were used. For comparison between the two groups, the Mann-Whitney test was used for continuous variables and chi-squared or Fisher's exact tests for dichotomous variables. Changes over time were analysed using Wilcoxon signed rank test for continuous variables and McNemar's test for dichotomous variables. The prognostic factors used in the univariate and multivariate analyses were data obtained from before anorexia nervosa onset or before Study 2. In the univariate analysis, for comparison between the two groups, Fisher's non-parametric permutation test was used. Formal testing of correlations was performed using Pitman's non-parametric permutation test and for descriptive purposes Pearson's correlation coefficient was calculated. All dependent variables were transformed to normal distribution using Blom's normal score transformation prior to multivariate analysis, which was performed with multiple stepwise linear regression analysis. Only variables with $P<0.15$ in univariate tests were included as possible predictors in the multiple stepwise procedure, owing to multiple testing at each step and to possible complex interrelationships among the variables. For survival analysis, the log-rank test was used for dichotomous variables and Cox's proportional hazard model for continuous and ordered categorical variables. Survival curves were estimated with the Kaplan-Meier method. In order to select independent predictors, stepwise Cox's proportional hazard regression was performed. Only variables with $P<0.15$ were included as possible predictors in the multivariate analysis. Hazard ratios (HRs) with 95\% confidence intervals (CIs) were calculated for descriptive purposes. All tests were two-tailed and conducted at a $5 \%$ significance level.

\section{Predictor variables}

The univariate and stepwise analyses of GAF and Morgan-Russell scores as dependent outcome variables were first conducted with variables measured at anorexia nervosa onset including retrospective childhood data (early gastrointestinal problems, problems with friends before the disorder onset, major problems in family before onset, major life events before onset, parental divorce before onset, death of parent or sibling before onset, premorbid affective disorder, premorbid overweight, premorbid social class, premorbid obsessive-compulsive disorder, premorbid obsessive-compulsive personality disorder, prenatal, perinatal and neonatal factors, premorbid BMI and age at disorder onset) and then with variables measured in Study 2 or before (i.e. variables measured at anorexia nervosa onset including premorbid factors): dysdiadochokinesia in Study 1 and/or Study 2, autism-spectrum condition before anorexia nervosa onset and/or in Study 2, restrictive subtype in Study 1 and Study 2, anxiety disorder in Study 1 and/or Study 2, obsessive-compulsive disorder in Study 1 and/or Study 2, full-scale IQ in Study 2 and minimum BMI in Study 2.

\section{Results}

The mean ages of the sample and the interval between anorexia nervosa onset and time of assessment in Study 4 are given in Table 1.

\section{Eating and other psychiatric disorders}

In Study 4, six women (12\%) still met the criteria for an eating disorder (Fig. 1). Altogether three women (6\%) had anorexia nervosa, all with a restrictive subtype. Anorexia nervosa from onset until Study 4 was present in only one woman. One woman had been free from anorexia nervosa and other eating disorders for several years, but had had a relapse of anorexia nervosa 6 months before Study 4. Another woman had had a diagnosis of EDNOS for several years, but in Study 4 she fulfilled the criteria for anorexia nervosa. Three women had EDNOS with bulimic behaviour. Body mass index values did not differ between the anorexia nervosa and comparison groups (anorexia nervosa group, mean $=22.4 \mathrm{~kg} / \mathrm{m}^{2}$, s.d. $=4.5$, range $13.6-37.7$; comparison group, mean $=23.4 \mathrm{~kg} / \mathrm{m}^{2}$, s.d. $=4.2$, range $18.0-38.1$ ).

\section{Full symptomatic recovery}

Overall, 27 (54\%) individuals in the anorexia nervosa group compared with $45(88 \%)$ individuals in the comparison group had been free from disturbed behaviour and attitudes with respect to food and shape in the past 6 months $(P<0.001)$. Eleven women $(22 \%)$ in the anorexia nervosa group were 'meat avoiders' (i.e. they were either vegetarians $(n=6)$ or just avoided meat $(n=5))$ $v$. one woman in the comparison group $(2 \%)(P<0.0001)$. Full

\begin{tabular}{|c|c|c|c|c|c|c|}
\hline & \multicolumn{3}{|c|}{ Anorexia nervosa group $(n=51)$} & \multicolumn{3}{|c|}{ Comparison group $(n=51)$} \\
\hline & Mean & Median & Range & Mean & Median & Range \\
\hline \multicolumn{7}{|l|}{ Age, years } \\
\hline Onset & 14.3 & 14.3 & $10.0-17.2$ & & & \\
\hline Study 1 & 16.1 & 16.2 & $11.1-19.1$ & 16.0 & 16.3 & $11.3-18.8$ \\
\hline Study 2 & 21.0 & 21.3 & $15.1-23.1$ & 20.8 & 21.1 & $14.9-22.8$ \\
\hline Study 3 & 24.5 & 25.0 & $18.2-26.8$ & 24.2 & 24.6 & $18.0-26.4$ \\
\hline Study 4 & 32.4 & 32.9 & $26.0-34.5$ & 32.4 & 33.0 & $26.2-34.9$ \\
\hline \multicolumn{7}{|l|}{ Time elapsed, years } \\
\hline Onset to Study 2 & 6.7 & 6.7 & $3.8-10.8$ & & & \\
\hline Onset to Study 3 & 10.2 & 10.3 & 7.0-13.9 & & & \\
\hline Onset to Study 4 & 18.1 & 18.3 & $14.6-21.5$ & & & \\
\hline
\end{tabular}


recovery occurred in $18 \%$ of the meat avoiders and in $63 \%$ of the 'non-meat avoiders' $(P=0.015)$. Thirty-nine per cent of the anorexia nervosa group $(n=20)$ met the criteria for at least one psychiatric disorder at follow-up (comparison group, $16 \% ; n=8$ ) $(P=0.004)$ (Table 2). The single most common psychiatric disorder was obsessive-compulsive disorder, affecting $16 \%(n=8)$ in the anorexia nervosa group and none in the comparison group $(P=0.006)$. Twenty-two per cent of the anorexia nervosa group $(n=11)$ had an affective disorder (comparison group, $4 \% ; n=2)$ $(P=0.015)$, with a concurrent eating disorder in only two cases.

\section{Life course of eating and other psychiatric disorders}

Figure 1 illustrates lifetime eating disorder diagnoses in the anorexia nervosa group. Only 12 individuals (24\%) in the anorexia nervosa group had never exhibited bulimic behaviour. The average duration of anorexia nervosa was 3.4 years (s.d.=2.4, range 0.9-14.7). The total duration of eating disorders (including the anorexia nervosa period) in the anorexia nervosa group was, on average, 7.5 years (s.d. $=4.7$, range $0.9-19.0$ ). In the anorexia nervosa group all but one had at some time met the criteria for an affective disorder (Table 2). Anxiety disorders had been significantly more common over the years in this group, affecting $73 \%(n=37)$ at some time compared with $39 \%(n=20)$ in the comparison group $(P=0.001)$. Among the anxiety disorders, obsessive-compulsive disorder was by far the most common diagnosis over the years, and lifetime prevalence differed significantly from the comparison group (anorexia nervosa group, $n=21$; comparison group, $n=5 ; P<0.0001$ ) (Table 2).

\section{Morgan-Russell scales}

According to the general outcome classification (only applied to the anorexia nervosa group), $6 \%(n=3)$ had a poor outcome, $10 \%(n=5)$ had an intermediate outcome and $84 \%(n=43)$ had good outcome. In terms of persistence of eating disorder, $12 \%$ $(n=6)$ had a poor outcome, including all cases with a current eating disorder, $10 \%(n=5)$ had intermediate outcome and $78 \%$ $(n=40)$ had a good outcome. The Morgan-Russell averaged scale scores were 9.7 (s.d. $=2.1$, range 3.6-12.0) and 11.0 (s.d.=1.2, range 5.6-12.0) for the anorexia nervosa and comparison groups respectively $(P<0.0001)$. Comparing the averaged Morgan-Russell scale scores with scores in our earlier studies, the anorexia nervosa group had scored significantly worse in Study 2 (8.7, s.d.=2.4, range $2.6-12.0 ; P<0.0001)$ but there was no significant improvement compared with Study 3 (9.4, s.d.=2.2, range 4.1-12.0; $P=0.058)$.

Thirteen $(25 \%)$ individuals in the anorexia nervosa group had no paid employment owing to their eating disorder and/or other psychiatric problems compared with one individual $(2 \%)$ in the comparison group $(P=0.001)$. All individuals with a persisting eating disorder had sickness pension or were on extended sick leave. The mean GAF score was significantly lower in the anorexia nervosa group: 65.2 (median=70, s.d. $=18.0$, range 30-90) compared with 82.4 (median=88; s.d. $=12.8$, range $30-95$ ) in the comparison group $(P<0.0001)$. Forty-one per cent in the anorexia nervosa group had poor psychosocial functioning with GAF scores of 60 or below. All the individuals with a persisting eating disorder belonged to this poor outcome group. Other

Table 2 Current and lifetime Axis I disorders in the anorexia nervosa and comparison groups

\begin{tabular}{|c|c|c|c|c|c|c|}
\hline & \multicolumn{4}{|c|}{ Current diagnoses } & \multicolumn{2}{|c|}{ Lifetime diagnoses } \\
\hline & \multicolumn{3}{|c|}{ Anorexia nervosa group } & \multirow{2}{*}{$\begin{array}{l}\text { Comparison } \\
\text { group } \\
\text { Total } \\
(n=51)\end{array}$} & \multirow{2}{*}{$\begin{array}{c}\text { Anorexia nervosa } \\
\text { group } \\
\text { Total } \\
(n=51)\end{array}$} & \multirow{2}{*}{$\begin{array}{c}\text { Comparison } \\
\text { group } \\
\text { Total } \\
(n=51)\end{array}$} \\
\hline & $\begin{array}{l}\text { No eating disorder } \\
\qquad(n=45)\end{array}$ & $\begin{array}{l}\text { Eating disorder } \\
\qquad(n=6)\end{array}$ & $\begin{array}{l}\text { Total } \\
(n=51)\end{array}$ & & & \\
\hline \multicolumn{7}{|l|}{$\begin{array}{l}\text { Affective disorders } \\
\text { Major depression }\end{array}$} \\
\hline Unipolar & 4 & 2 & 6 & 0 & 34 & 12 \\
\hline Bipolar I & 2 & 0 & 2 & 1 & 3 & 1 \\
\hline Bipolar II & 2 & 0 & 2 & 1 & 2 & 1 \\
\hline Dysthymic disorder & 1 & 0 & 1 & 0 & 11 & 2 \\
\hline Any affective disorder & 9 & 2 & $11 *$ & 2 & $50 * * *$ & 16 \\
\hline \multicolumn{7}{|l|}{ Anxiety disorders } \\
\hline Panic disorder & 1 & 1 & 2 & 1 & 8 & 4 \\
\hline Agoraphobia & 0 & 1 & 1 & 0 & 1 & 0 \\
\hline Social phobia & 0 & 1 & 1 & 0 & 3 & 1 \\
\hline Specific phobia & 1 & 1 & 2 & 3 & 9 & 10 \\
\hline Obsessive-compulsive disorder & 5 & 3 & $8 * *$ & 0 & $21 * * *$ & 5 \\
\hline Post-traumatic stress disorder & 1 & 0 & 1 & 1 & 1 & 1 \\
\hline General anxiety disorder & 3 & 2 & 5 & 1 & 15 & 8 \\
\hline Any anxiety disorder & 10 & 4 & 14 & 6 & $37 * *$ & 20 \\
\hline Any anxiety disorder ${ }^{a}$ & 5 & 4 & 9 & 6 & 26 & 18 \\
\hline \multicolumn{7}{|l|}{ Psychotic disorders } \\
\hline Paranoid schizophrenia & 1 & 0 & 1 & 1 & 1 & 1 \\
\hline Schizophreniform disorder & 1 & 0 & 1 & 0 & 1 & 0 \\
\hline Psychosis NOS & 0 & 0 & 0 & 0 & 1 & 0 \\
\hline Any psychotic disorder & 2 & 0 & 2 & 1 & 3 & 1 \\
\hline \multicolumn{7}{|l|}{ Impulse control disorders } \\
\hline Substance misuse & 0 & 0 & 0 & 0 & 2 & 3 \\
\hline Any Axis I disorder ${ }^{b}$ & 16 & 4 & $20 * \star$ & 8 & $51 * * *$ & 29 \\
\hline \multicolumn{7}{|c|}{$\begin{array}{l}\text { NOS, not otherwise specified. } \\
\text { a. Excluding obsessive-compulsive disorder. } \\
\text { b. Excluding eating disorders. } \\
{ }^{*} P<0.05 ;{ }^{* *} P<0.01 ;{ }^{* * *} P<0.0001 \text {; all anorexia nervosa group } v \text {. comparison group. }\end{array}$} \\
\hline
\end{tabular}


individuals with low GAF scores had one or more Axis I disorders and/or severe problems with regard to social contacts.

Thirty-one individuals (61\%) had received psychotherapy, defined as child or adult psychiatric treatment based on psychodynamic interventions given on eight occasions or more. Ten individuals $(20 \%)$ had either consulted psychiatric services on fewer than eight occasions or had been treated by a non-specialist. Ten individuals (20\%) had never consulted anyone for their eating disorder or for any other psychiatric disorder. There was no significant difference regarding GAF and Morgan-Russell scale scores in Study 4 among those who had received treatment at all and those who had not. The 31 individuals who had received psychotherapy had a longer duration of anorexia nervosa $(4.40$ years (s.d.=3.0) v. 2.31 years (s.d. $=1.2) ; P=0.002)$, lower GAF score (60.0 (s.d.=18.7) v. 73.2 (s.d.=13.7); $P=0.014)$, lower age at anorexia nervosa onset (13.8 years (s.d.=1.6) v. 14.9 years (s.d.=1.2); $P=0.027)$ and lower minimum BMI ever $\left(14.4 \mathrm{~kg} / \mathrm{m}^{2}(\right.$ s.d. $=2.7)$ v. $16.0 \mathrm{~kg} / \mathrm{m}^{2}$ (s.d.=2.0); $P=0.032$ ) compared with the rest of the anorexia nervosa group.

\section{Predictive factors}

Significant predictors and tendencies in the univariate analysis of GAF score as dependent outcome variable were early gastrointestinal problems $(P=0.066)$, age at anorexia nervosa onset $(P=0.070)$, autism-spectrum condition before anorexia nervosa onset and/or in Study $2(P=0.007)$ and minimum BMI ever in Study $2(P=0.043)$. Significant predictors and tendencies in the univariate analysis of Morgan-Russell score as dependent outcome variable were problems with friends before anorexia nervosa onset $(P=0.096)$, premorbid obsessive-compulsive disorder $(P=0.079)$, premorbid obsessive-compulsive personality disorder $(P=0.019)$, age at anorexia nervosa onset $(P=0.092)$, dysdiadochokinesia in Study 1 and/or Study $2(P=0.033)$ and autism-spectrum condition before anorexia nervosa onset and/ or in Study $2(P=0.031)$.

\section{Stepwise analysis}

Independent predictors, measured in Study 2 or before, of poor outcome according to GAF in Study 4 were autism-spectrum condition before anorexia nervosa onset and/or in Study 2 $(P=0.0023)$ and age at anorexia nervosa onset (the lower the age, the lower the GAF score) $(P=0.021)$ (model $\left.r^{2}=0.29\right)$. Independent predictor of poor outcome according to Morgan-Russell scores in Study 4 among variables measured before anorexia nervosa onset was premorbid obsessive-compulsive personality disorder $(P=0.016)$ (model $\left.r^{2}=0.11\right)$ and independent predictors among variables measured in Study 2 or before were premorbid obsessive-compulsive personality disorder $(P=0.016)$, autismspectrum condition before anorexia nervosa onset and/or in Study $2(P=0.034)$ and age at onset (the lower the age, the lower the Morgan-Russell score) $(P=0.047)$ (model $\left.r^{2}=0.26\right)$.

\section{Survival analysis}

Table 3 shows the results for the survival analysis, using the duration of the first anorexia nervosa episode as the dependent outcome variable. The multivariate analysis rendered two significant independent predictors, namely age at anorexia nervosa onset (the lower the age, the longer the duration of the first anorexia nervosa episode $)(P<0.0009$; HR 1.52, 95\% CI 1.19-1.94) and premorbid obsessive-compulsive personality disorder $(P<0.0091$; $\mathrm{HR}=0.41,95 \%$ CI $0.21-0.80$ ).

\section{Discussion}

Long-term follow-up studies are extremely important in anorexia nervosa, considering the lengthy duration of the illness in many cases. In this study the mean duration was over 3 years. Furthermore, the anorexia nervosa group fulfilled the diagnoses of bulimia nervosa or EDNOS for another 4 years on average. In addition, in an adolescent sample a longer follow-up time is needed to evaluate the impact of the eating disorder on adult life, with challenges such as getting a job, finding a partner and having children.

The strengths of our study include its prospective longitudinal approach. It is the first long-term follow-up study of anorexia nervosa with a community-based sample. ${ }^{9}$ The sample included only individuals with teenage onset of anorexia nervosa. A matched comparison group with no eating disorder was assessed at the same time and with the same instruments in the original study and at follow-up. There was no attrition.

No death has occurred in either sample during the 18 years that we have followed the 51 participants with anorexia nervosa and the 51 individuals in the matched comparison group. This is a better result than in other long-term follow-up studies, which have reported a mortality rate of almost $20 \% .^{2-4}$ In his review of follow-up studies, Steinhausen concluded that mortality rates increased significantly with the length of the follow-up. ${ }^{1}$ Another Swedish study, based on registers, reported a low mortality rate: $1.2 \%$ at 9-14-year follow-up of adolescent in-patients with anorexia nervosa. ${ }^{18}$ It would seem that recent Swedish outcome studies have a much better prognosis in comparison with other countries' results. ${ }^{8,18}$ However, these Swedish studies are in agreement with other follow-up studies with teenage-onset anorexia nervosa, in which low or zero mortality rates have been reported. ${ }^{5,6}$ The results may be due to better treatment strategies in recent years and may apply to older patients as well.

At the 18-year follow-up only six individuals had persisting eating disorder; three of whom still had anorexia nervosa. This

\begin{tabular}{|c|c|c|}
\hline Predictor & $\mathrm{HR}(95 \% \mathrm{Cl})$ & $P^{a}$ \\
\hline Prenatal, perinatal and neonatal factors ${ }^{\mathrm{b}}$ & $1.05(0.88-1.26)$ & 0.58 \\
\hline Early gastrointestinal problems & $0.99(0.56-1.73)$ & 0.97 \\
\hline Problems with friends before onset & $0.76(0.43-1.34)$ & 0.35 \\
\hline Major problems in family before onset & $1.33(0.70-2.53)$ & 0.38 \\
\hline Major life events before onset & $1.17(0.66-2.08)$ & 0.58 \\
\hline Parental divorce before onset & $1.14(0.63-2.07)$ & 0.67 \\
\hline Death of parent or sibling before onset & $1.14(0.53-2.45)$ & 0.74 \\
\hline Premorbid affective disorder & $1.14(0.41-3.21)$ & 0.80 \\
\hline Premorbid BMI & $1.13(1.04-1.23)$ & 0.003 \\
\hline Premorbid overweight & $1.41(0.70-2.85)$ & 0.34 \\
\hline Premorbid social class 1 & $1.25(0.30-5.19)$ & 0.76 \\
\hline Premorbid social class 2 & $0.76(0.42-1.37)$ & 0.36 \\
\hline Premorbid social class 3 & $1.27(0.71-2.26)$ & 0.42 \\
\hline Premorbid OCD & $0.66(0.33-1.31)$ & 0.23 \\
\hline Premorbid OCPD & $0.61(0.33-1.11)$ & 0.11 \\
\hline Age at onset & $1.35(1.08-1.69)$ & 0.008 \\
\hline Primary amenorrhoea ${ }^{\mathrm{C}}$ & $1.82(0.77-4.34)$ & 0.17 \\
\hline \multicolumn{3}{|c|}{$\begin{array}{l}\text { BMI, body mass index; HR, hazard ratio; OCD, obsessive-compulsive disorder; } \\
\text { OCPD, obsessive-compulsive personality disorder. } \\
\text { a. Cox regression model. } \\
\text { b. Based on data from } 47 \text { individuals. } \\
\text { c. Based on data from the } 48 \text { female participants. }\end{array}$} \\
\hline
\end{tabular}


finding is one indication of a favourable long-term outcome and is consistent with recent research focusing on teenage anorexia nervosa. ${ }^{5,6,8,19}$ On the other hand, as in Strober et al's teenage sample, ${ }^{5}$ symptoms pertaining to over-awareness of body shape, weight and tension at mealtimes tended to persevere. These are problems that, in turn, have implications for social life, and may contribute to the poor psychosocial outcome in anorexia nervosa.

In our study, $39 \%$ had a psychiatric disorder other than an eating disorder. One in five had an affective disorder. This is in line with earlier outcome studies. ${ }^{1,6,20}$ In our study the rate of psychiatric comorbidity had not changed since our previous follow-up, 10 years after the onset of anorexia nervosa. ${ }^{7}$ However, in that study we concluded that affective disorders seemed to subside when the individuals had recovered from their eating disorder. In this latest assessment, 11 individuals in the anorexia nervosa group had an affective disorder and only 2 of them had a persisting eating disorder. Thus, we found substantial problems with affective disorders even after the individuals had recovered from their eating disorder.

Our teenage-onset, community-based anorexia nervosa group had a better outcome of eating disorder per se compared with clinical samples. Nevertheless, we did not find the expected better outcome with respect to employment and psychiatric symptoms. ${ }^{3,19,21}$ In young adults in the anorexia nervosa group, one in four had no paid employment as a consequence of psychiatric problems that included eating disorders. All individuals with a persisting eating disorder were unemployed. It has not previously been observed that so many individuals with teenage-onset anorexia nervosa remain unemployed owing to persisting psychiatric morbidity almost two decades after illness onset.

Sixty-one per cent of the anorexia nervosa group had received psychotherapy. These individuals had longer duration of anorexia nervosa, lower GAF scores in Study 4, lower age at onset and lower minimum BMI ever. As this is not a treatment study it is difficult to draw any conclusions from these data. Individuals with a more severe form of eating disorder and co-existing psychiatric disorders would seem to be more likely to seek psychotherapeutic treatment at some point. At the time of the original study the reason for not seeking help was lack of opportunity. However, the research team referred those without psychiatric contacts. Nevertheless, many individuals and their families did not attend psychiatric services. Those who came to medical attention were generally not worse off than the rest. ${ }^{22}$ In the present community-based study, 20\% had never consulted anyone for their eating disorder or any other psychiatric disorder. Thus, in non-community-based treatment studies of anorexia nervosa, one should consider that one in five may never seek treatment.

\section{Predictive factors}

In this study we had access to a vast amount of data for this cohort from birth onwards. In Study 1, our group collected information meticulously on environmental background factors. ${ }^{23}$ Familial and other environmental factors before anorexia nervosa onset did not turn out to be predictors of importance with respect to outcome. Low age at anorexia nervosa onset in our study predicted unfavourable outcome according to all our outcome variables, consistent with a study focusing on a sample with younger individuals. ${ }^{24}$ Premorbid obsessive-compulsive personality disorder predicted poor outcome with respect to the duration of the first anorexia nervosa episode and Morgan-Russell scores. The diagnosis of premorbid obsessive-compulsive personality disorder had been assigned by a masked rater in Study 1 based on retrospective information from a collateral interview with the mother. According to two reviews there is reasonable evidence that this disorder is associated with a poor outcome. ${ }^{1,25}$ Autistic traits occurring premorbidly and/or in late adolescence or early adult life have not previously been reported as an unfavourable prognostic factor. Autism-spectrum conditions in childhood reported in Study 1 and/or in Study 2 predicted a poor outcome according to the global functioning scales used in this study. In Study 1 and Study 2, an autism-spectrum condition was assigned by a masked rater. We believe that other researchers who have described poor social skills and obsessionality before the onset of anorexia nervosa have probably come across individuals with a disorder belonging to the autism spectrum. ${ }^{5}$

\section{Limitations}

We have assessed our sample on only four separate occasions. The retrospective data, concerning among other things relapses between studies, may be less reliable. Our sample size is relatively small in comparison with other follow-up studies. ${ }^{2,5,26}$ The design of the original study, recruiting a homogeneous sample from the community and not from clinics, limited the number of available participants.

\section{Clinical implications}

To summarise, 18 years after the onset of anorexia nervosa in adolescence, the outcome regarding mortality and eating disorders was good. In terms of psychiatric and psychosocial outcomes the results were more discouraging, with 39\% experiencing other psychiatric disorders and one in four unable to work. Low age at anorexia nervosa onset, obsessive-compulsive personality disorder and autistic traits predicted an unfavourable outcome.

Elisabet Wentz, MD, PhD, Institute of Neuroscience and Physiology, Child and Adolescent Psychiatry, Göteborg University, and Vårdal Institute, Swedish Institute for Health Sciences, Sweden; I. Carina Gillberg, MD, PhD, Institute of Neuroscience and Physiology, Child and Adolescent Psychiatry, Göteborg University, Sweden; Henrik Anckarsäter, MD, PhD, Institute of Clinical Sciences, Lund University, Malmö, Sweden; Christopher Gillberg, MD, PhD, Institute of Neuroscience and Physiology, Child and Adolescent Psychiatry, Göteborg University, Sweden, and Department of Child and Adolescent Psychiatry, Strathclyde University, Glasgow, UK; Maria Råstam $\mathrm{MD}, \mathrm{PhD}$, Institute of Neuroscience and Physiology, Child and Adolescent Psychiatry, Göteborg University, and Institute of Clinical Sciences, Child and Adolescent Psychiatry, Lund University, Lund, Sweden

Correspondence: Dr Elisabet Wentz, Institute of Neuroscience and Physiology, Child and Adolescent Psychiatry, Göteborg University, Otterhällegatan 12 B, SE-411 18 Göteborg, Sweden. Email: elisabet.wentz@vgregion.se

First received 12 Dec 2007, final revision 22 Aug 2008, accepted 22 Sep 2008

\section{Acknowledgements}

Birgitta Melander was instrumental in assuring coverage of the original cohort of probands and controls. Independent statistical analysis was performed by Nils-Gunnar Pehrsson and Mikael Holtenman. This work was supported by the Swedish Research Council (K2006-21X20048-01-2), government grants under the Avtal om Läkarutbildning och Forskning (ALF) 20048-01-2), government grants under the Avtal om Läkarutbildning och Forskning (ALF)
Agreement on Medical Education and Research, Knut and Alice Wallenberg's Foundation, the Söderström Königska Nursing Home Foundation, the Swedish Medical Society and the Göteborg Freemasons.

\section{References}

1 Steinhausen HC. The outcome of anorexia nervosa in the 20th century. Am J Psychiatry 2002; 159: 1284-93.

2 Theander S. Outcome and prognosis in anorexia nervosa and bulimia: some results of previous investigations, compared with those of a Swedish longterm study. J Psychiatr Res 1985; 19: 493-508.

3 Ratnasuriya RH, Eisler I, Szmukler Gl, Russell GF. Anorexia nervosa: outcome and prognostic factors after 20 years. Br J Psychiatry 1991; 158: 495-502. 
4 Zipfel S, Lowe B, Reas DL, Deter HC, Herzog W. Long-term prognosis in anorexia nervosa: lessons from a 21-year follow-up study. Lancet 2000; 355 721-2.

5 Strober $\mathrm{M}$, Freeman R, Morrell $\mathrm{W}$. The long-term course of severe anorexia nervosa in adolescents: survival analysis of recovery, relapse, and outcome predictors over 10-15 years in a prospective study. Int J Eat Disord 1997; 22 $339-60$

6 Herpertz-Dahlmann B, Muller B, Herpertz S, Heussen N, Hebebrand J, Remschmidt H. Prospective 10-year follow-up in adolescent anorexia nervosa - course, outcome, psychiatric comorbidity, and psychosocial adaptation. J Child Psychol Psychiatry 2001; 42: 603-12.

7 Wentz E, Gillberg C, Gillberg IC, Rastam M. Ten-year follow-up of adolescentonset anorexia nervosa: psychiatric disorders and overall functioning scales. J Child Psychol Psychiatry 2001; 42: 613-22.

8 Nilsson K, Hägglöf B. Long-term follow-up of adolescent onset anorexia nervosa in Northern Sweden. Eur Eat Disord Rev 2005; 13: 89-100.

9 Berkman ND, Lohr KN, Bulik CM. Outcomes of eating disorders: a systematic review of the literature. Int J Eat Disord 2007; 40: 293-309.

10 Rastam M, Gillberg C, Garton M. Anorexia nervosa in a Swedish urban region. A population-based study. Br J Psychiatry 1989; 155: 642-6.

11 Rastam M. Anorexia nervosa in 51 Swedish adolescents: premorbid problems and comorbidity. J Am Acad Child Adolesc Psychiatry 1992; 31: 819-29.

12 American Psychiatric Association. Diagnostic and Statistical Manual of Mental Disorders (3rd edn, revised) (DSM-III-R). American Psychiatric Press, 1987.

13 American Psychiatric Association. Diagnostic and Statistical Manual of Mental Disorders (4th edn) (DSM-IV). American Psychiatric Press, 1994.

14 Gillberg IC, Rastam M, Gillberg C. Anorexia nervosa 6 years after onset: part I. Personality disorders. Compr Psychiatry 1995; 36: 61-9.

15 Gillberg IC, Gillberg C, Rastam M, Johansson M. The cognitive profile of anorexia nervosa: a comparative study including a community-based sample. Compr Psychiatry 1996; 37: 23-30.
16 First MB SR, Gibbon M, Williams JBW. Structured Clinical Interview for DSM-IV Axis I Disorders - Patient Edition (SCID-I/P, Version 2.0, 4/97 revision). New York Biometrics Research Department, 1997.

17 Morgan HG, Russell GF. Value of family background and clinical features as predictors of long-term outcome in anorexia nervosa: four-year follow-up study of 41 patients. Psychol Med 1975; 5: 355-71.

18 Hjern A, Lindberg L, Lindblad F. Outcome and prognostic factors for adolescent female in-patients with anorexia nervosa: 9- to 14-year follow-up. Br J Psychiatry 2006; 189: 428-32.

19 Steinhausen HC, Seidel R, Winkler Metzke C. Evaluation of treatment and intermediate and long-term outcome of adolescent eating disorders. Psychol Med 2000; 30: 1089-98

20 Fichter MM, Quadflieg N, Hedlund S. Twelve-year course and outcome predictors of anorexia nervosa. Int J Eat Disord 2006; 39: 87-100.

21 Lowe B, Zipfel S, Buchholz C, Dupont Y, Reas DL, Herzog W. Long-term outcome of anorexia nervosa in a prospective 21-year follow-up study. Psychol Med 2001; 31: 881-90.

22 Gillberg C, Rastam M, Gillberg IC. Anorexia nervosa: who sees the patients and who do the patients see? Acta Paediatr 1994; 83: 967-71.

23 Rastam M, Gillberg C. Background factors in anorexia nervosa. A controlled study of 51 teenage cases including a population sample. Eur Child Adolesc Psychiatry 1992; 1: 53-64.

24 Bryant-Waugh R, Knibbs J, Fosson A, Kaminski Z, Lask B. Long term follow up of patients with early onset anorexia nervosa. Arch Dis Child 1988; 63: 5-9.

25 Crane AM, Roberts ME, Treasure J. Are obsessive-compulsive personality traits associated with a poor outcome in anorexia nervosa? A systematic review of randomized controlled trials and naturalistic outcome studies. Int J Eat Disord 2007; 40: 581-8.

26 Sullivan PF, Bulik CM, Fear JL, Pickering A. Outcome of anorexia nervosa: a case-control study. Am J Psychiatry 1998; 155: 939-46. 